\title{
Evaluación de sistemas eólicos en el Caribe ${ }^{*}$
}

\section{Guillermo Becerra ${ }^{1}$ \\ José Hernández² \\ Edith Osorio ${ }^{1}$ \\ Jorge 0. Aguilar ${ }^{2}$ \\ Javier Vazquez ${ }^{2}$}

\author{
${ }^{1}$ Consejo Nacional de Ciencia y Tecnología \\ CONACYT-Universidad de Quintana Roo \\ ¿Universidad de Quintana Roo \\ División de Ciencias e Ingeniería \\ Boulevar Bahía s/n, Chetumal, CP 77019, Quintana Roo. \\ MÉXICO
}

trabajo se describen algunos modelos para la representación de aerogeneradores, experimentos mediante un túnel de viento con una turbina de viento y el recurso eólico local.

Palabras clave: energía limpia, turbina de viento, túnel de viento, potencia eólica.

\section{Abstract \\ (Evaluation of Wind Systems in the Caribbean)}

Economic growth is strongly linked to energy consumption, as well as to polluting emissions, however the transformation to electricity, through fossil sources, it has left an important mark on the planet. Of the main alternatives in recent years, great potential has been devoted to studying and taking advantage of the energy coming from the sun, as well as the use of wind, which finally is another transformation of the solar energy absorption. On the other hand, the efficient use of wind energy has multiple challenges to solve as a source of electricity generation and, especially at the University of Quintana Roo, different aspects are studied, to achieve its use with the mayor benefit from the aforementioned source of clean energy. In the present work, some models for the representation of wind turbines, experiments through a wind tunnel with an aerogenerator and the local wind resource are presented.

Index terms: clean energy, wind turbine, wind tunnel, wind power.

\section{Introducción}

La energía que proviene del viento se ha aprovechado desde muchos años atrás. Tradicionalmente se han empleado molinos de viento para obtener energía mecánica, en la antigüedad los principales usos se enfocan al bombeo de agua y molinos de granos. Sin embargo, como se menciona en Chaves [1], en la revolución industrial por la aparición de la máquina de vapor, los molinos de viento pierden importancia, pero desde la crisis del petróleo se retoma y, en general, la industria de la energía eólica se ha centrado en la generación de electricidad a partir de grandes aerogeneradores ubicados en lugares expuestos como: montañas y costas, con velocidades del viento medias altas [2], [3], lo cual, consiste en ofrecer energía renovable a costo competitivo con el mercado.

Como ejemplo principal en México se pueden resaltar los aerogeneradores instalados en el Istmo de Tehuantepec, 
Oaxaca, donde se han instalado dispositivos de gran escala para la red eléctrica del país [4], [5], [6]. Sin embargo, a nivel mundial existen múltiples instalaciones, de las que se pueden resaltar hasta 2017 [7], los principales productores por región: Sudáfrica 2,094 MW (África), China 188,232 MW (Asia), Alemania 56,132 MW (Europa), Brasil 12,763 MW (Latinoamérica y el Caribe), USA 89,077 MW (Norteamérica); Cabe resaltar que México produce 4,005 MW.

Por otro lado, recientemente, han aumentado los fabricantes de pequeñas turbinas, principalmente para usos particulares. Aunque la definición de sistemas eólicos pequeños generalmente se acepta para incluir cualquier dispositivo con una potencia menor a $50 \mathrm{~kW}$, las turbinas para aplicaciones domésticas generan 2 o $3 \mathrm{~kW}$ de potencia. Se pueden montar en techos o postes independientes y generalmente se conectan al tablero de distribución del usuario [8]. La electricidad que se genera puede ser usada directamente en el sitio, y cualquier excedente se ingresa a la red eléctrica. Para alcanzar dichas potencias de generación eléctrica, generalmente son turbinas de arranque con velocidad de viento nominal mayor a $10 \mathrm{~m} / \mathrm{s}$ instaladas a una altura por arriba de $10 \mathrm{~m} 10 \mathrm{~m}$ sobre el nivel del mar [6].

Por otro lado, en Soler-Bientz et al. [9] de 2000 - 2007, para la península de Yucatán, Hernández- Escobedo et al. [10] 2000 2008, para todo México, reportan las velocidades del viento promedio, donde se puede notar que, para la ciudad de Chetumal, perteneciente al Caribe Mexicano, el promedio mencionado, está por debajo de $5 \mathrm{~m} / \mathrm{s}$. Los datos son obtenidos cada 10 minutos por las estaciones meteorológicas respectivas, con los que se calculan los promedios. Sin embargo, algunos estudios cercanos a la región, como el caso de la isla de Cozumel [11], donde se describe la posibilidad de instalación de un sistema eólico en combinación con fotovoltaico, apoyaría la reducción del costo y emisiones de $\mathrm{CO}_{2}$. Además, en [12], Hernández-Gálvez et al. presentan la investigación y caso de estudio para algunos sitios del estado de Tabasco México, donde existe la posibilidad de implementar sistemas eólicos en lugares de bajo recurso, que normalmente se descartan por el costo beneficio.

Por lo que es importante realizar un estudio en el diseño de los aerogeneradores para evaluar y en principio, aprovechar el recurso eólico en sitios donde la velocidad del viento promedia menos de $10 \mathrm{~m} / \mathrm{s}$. El presente trabajo hace un análisis de energía eólica en la zona, así como experimentos con un túnel de viento y aerogenerador para determinar su captación de energía.

El resto del documento está organizado de la siguiente forma, en la sección 2 se describen los aspectos teóricos para el modelo del sistema de generación a partir de la energía del viento, la sección 3 describe los sistemas eólicos utilizados para los experimentos y los perfiles del recurso eólico local. La sección 4 describe los experimentos realizados con los mencionados sistemas y la sección 5 finaliza con la discusión y conclusiones del trabajo.

\section{Aspectos teóricos del sistema}

Del aprovechamiento de energía eólica, que hoy en día se transforma a electricidad, al hacer girar una turbina mediante un ingenioso diseño. En esta sección se presentan algunos modelos matemáticos, para describir el comportamiento de este sistema, que normalmente es empleado en la literatura.

\subsection{Potencia eólica-mecánica}

Se han empleado diferentes enfoques teóricos para describir la transformación de energía que proviene del viento, algunos de los cuales se describen en [3], [4], [13], [14], [15], [16], [17], [18], [19], [20], entre otros.

Donde un modelo usual de potencia $(P)$, en términos del coeficiente de potencia $\left(C_{p}\right)$, que indica la eficiencia de conversión entre la potencia eólica - mecánica del aerogenerador es:

$$
P=0.5 \rho C_{p}(\lambda, \alpha) A_{R} V_{v}^{3}
$$

donde $\rho$ es la densidad del aire, $A_{R}$ el área cubierta por los álabes durante la rotación, $V_{v}$ la velocidad del viento y

$$
\begin{gathered}
C_{p}=0.5\left[\frac{116}{\lambda_{i}}-0.4 \alpha-5 e\right]^{\frac{-21}{\lambda i}} \\
\frac{1}{\lambda_{i}}=\frac{1}{\lambda+0.08 \alpha}-\frac{0.35}{\alpha^{3}+1}, \quad \lambda=\frac{R \omega}{V_{v}}
\end{gathered}
$$

con $\lambda$ la relación de velocidad de la punta del álabe, $\alpha$ el ángulo de inclinación del álabe, $R$ el radio del álabe, $\omega$ la velocidad angular del rotor de la turbina.

Además, la relación general de $C_{p}$, para el análisis de aerogenerador o ventilador, puede ser expresada de la forma,

$$
C_{p}=\operatorname{sign}\left(\lambda^{\prime}\right) 0.5\left[\frac{116}{\lambda_{i}}-0.4 \alpha-5 e\right]^{\frac{-21}{\lambda_{i}}}
$$

donde $\lambda$ ' es la velocidad relativa normalizada, 


$$
\lambda^{\prime}=\frac{V_{v}-r \omega}{V_{m}}
$$

con $r$ como el radio equivalente $(r \neq R), V_{m}$ la velocidad media del viento.

Mediante las ecuaciones anteriores se puede graficar el coeficiente de potencia, con respecto a la velocidad relativa normalizada, para la posibilidad de operación del generador ante diferentes $\alpha$, (véase Fig. 1).

Por otro lado, en diferentes trabajos [18], [19], [20] se analizan otros detalles de la generación como los efectos del generador eléctrico acoplado y la electrónica de potencia utilizada para regular los efectos variantes.

Con lo que se define la potencia extraíble del viento $P_{v}\left[\mathrm{~kW} / \mathrm{m}^{2}\right]$,

$$
P_{V}=\left[\begin{array}{cr}
0 & V_{V}<V_{c i} \\
P & V_{c i} \leq V_{V}<V_{n} \\
P_{n} & V_{n} \leq V_{V}<V_{c f} \\
0 & V_{c f} \leq V_{V}
\end{array}\right]
$$

donde $P_{n}$ es la potencia nominal, $V_{c i}$ la velocidad de arranque (inicial), $V_{n}$ la velocidad nominal, $V_{c f}$ la velocidad de paro (final). Lo cual capta la energía en un rango de velocidad [21], como se muestra en la Fig. 2, de forma dinámica antes de la nominal.

\section{Sistemas eólicos}

Se realizaron experimentos con la turbina eólica (Air X $400 \mathrm{~W}$ ) de wind power Manual AIR-X [21], y el túnel de viento (Subsonic), con los cuales se cuenta en la Universidad de Quintata Roo.

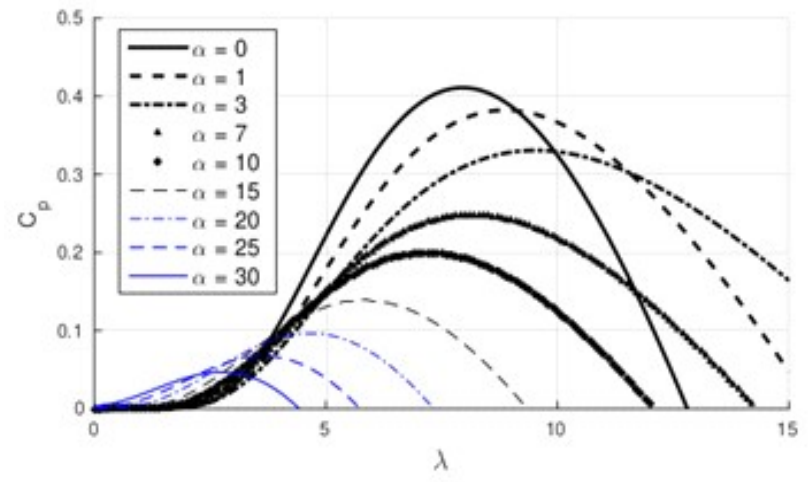

Fig. 1. Curvas características $C_{p}$ vs $\lambda$ del aerogenerador

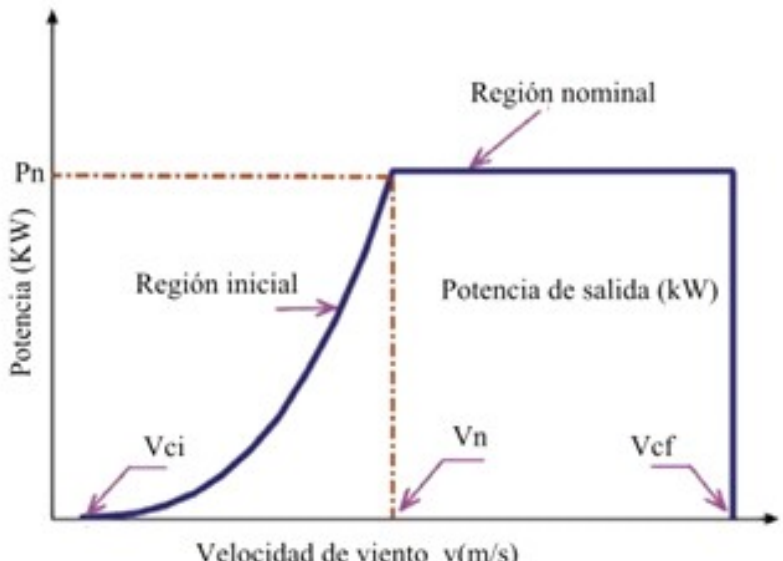

Fig. 2. Curva característica de potencia de turbina de viento.

\section{Desarrollo del problema}

El túnel de viento subsónico o túnel aerodinámico es una herramienta que se utiliza normalmente en mecánica de fluidos para diferentes experimentos, el cual, tiene la opción de aplicar diferentes velocidades de viento en sistemas a escala tales como edificios, aeronaves, puentes, vehículos, turbinas de viento (como la del presente trabajo), entre otros.

La Fig. 3 muestra el túnel y aerogenerador que se emplearon en el experimento, el aire ingresa por un alisador de flujo de nido de abeja de aluminio (lado izquierdo), diseñado para garantizar que el flujo sea constante tanto en magnitud como en dirección, al continuar, el aire atraviesa la sección de trabajo (ancho $304 \mathrm{~mm} \times$ alto $304 \mathrm{~mm} \times$ largo $457 \mathrm{~mm}$ ) y sale del túnel extraído por un ventilador de 5 álabes, que gira un motor de AC con frecuencia variable, para controlar la velocidad. En el lado derecho de la figura, se aprecia la turbina eólica.

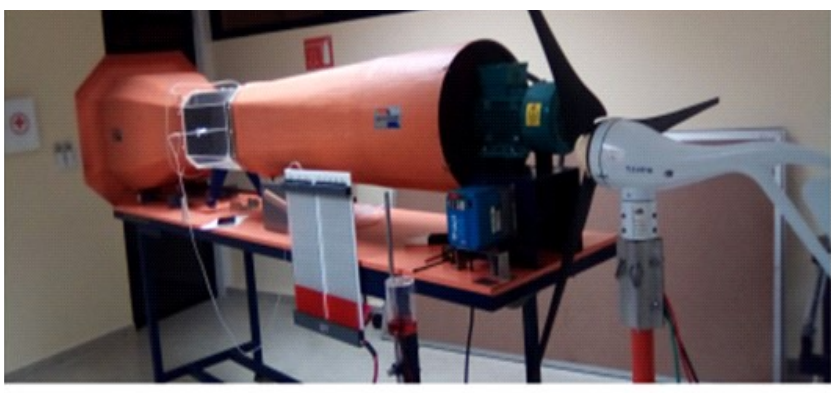

Fig. 3. Montaje del túnel de viento sobre la mesa de trabajo (equipo naranja) y turbina eólica Air X (carcaza blanca, con aspas negras). 


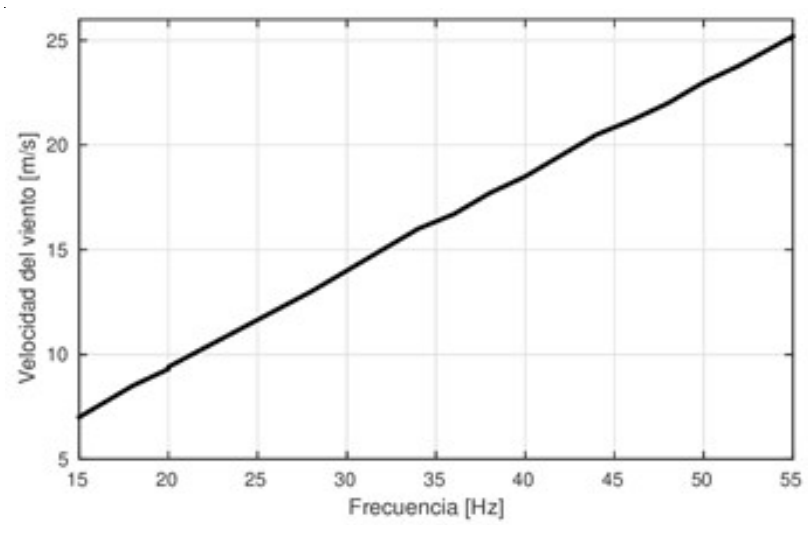

Fig. 4. Incremento de la velocidad del viento en el área de trabajo del túnel de viento, ante incremento de frecuencia en el variador de velocidad del motoventilador.

La Fig. 4 muestra el aumento de frecuencia en el variador de velocidad, para el motor de AC que acciona el ventilador y extrae el viento. Al aumentar la frecuencia, se incrementa la velocidad del viento en el área de trabajo del túnel de viento hasta un valor cercano a $\operatorname{los} 25 \mathrm{~m} / \mathrm{s}$.

Sin embargo, por las dimensiones internas del área de trabajo del túnel $\left(A_{T}=0.092 \mathrm{~m}^{2}\right)$, no es posible ingresar el aerogenerador con área de $\left(A_{A}=1.075 \mathrm{~m}^{2}\right)$, para el experimento con viento homogéneo. Por lo tanto, se experimentó colocando la turbina eólica a $70 \mathrm{~cm}$ de distancia en la descarga del túnel, donde se midieron las velocidades de viento a la salida del túnel. Primero se analizó el mapa de velocidades del viento para un radio de $40 \mathrm{~cm}$ desde el centro del túnel, para diferentes valores de frecuencia (véase Fig. 5), donde se puede ver que las velocidades del viento son muy diferentes de

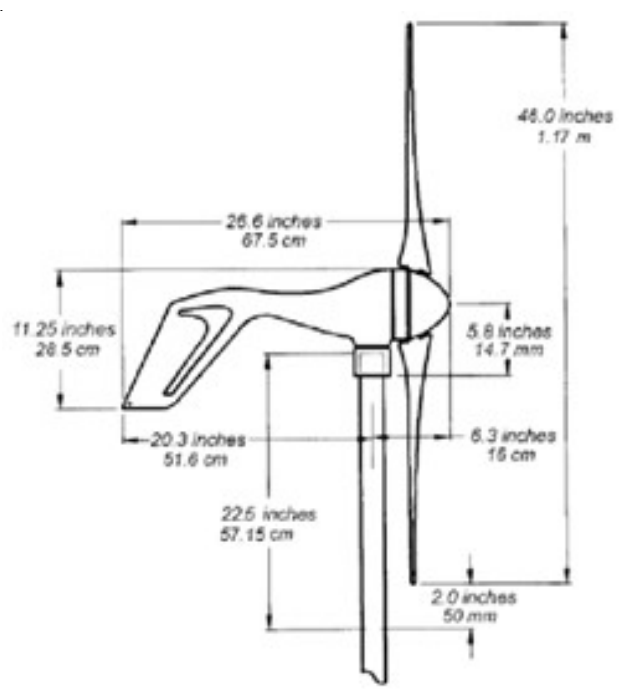

Fig. 6. Dimensiones geométricas del aerogenerador.

acuerdo con el punto elegido y se debe a la descarga del túnel. Además, la orientación del viento ya no se controla, lo que se aproxima a condiciones más reales para los experimentos con la turbina eólica.

\subsection{Turbina eólica}

De las características principales en cuanto al aerogenerador, de acuerdo con el fabricante, el peso de la turbina es $6[\mathrm{~kg}]$, el área de esta durante la rotación es de 1.075 [m], los detalles geométricos se muestran en la Fig. 6.

La velocidad del viento para el arranque es de $3.0[\mathrm{~m} / \mathrm{s}]$ y la nominal de $12[\mathrm{~m} / \mathrm{s}]$, lo que sería el punto ideal de operación.
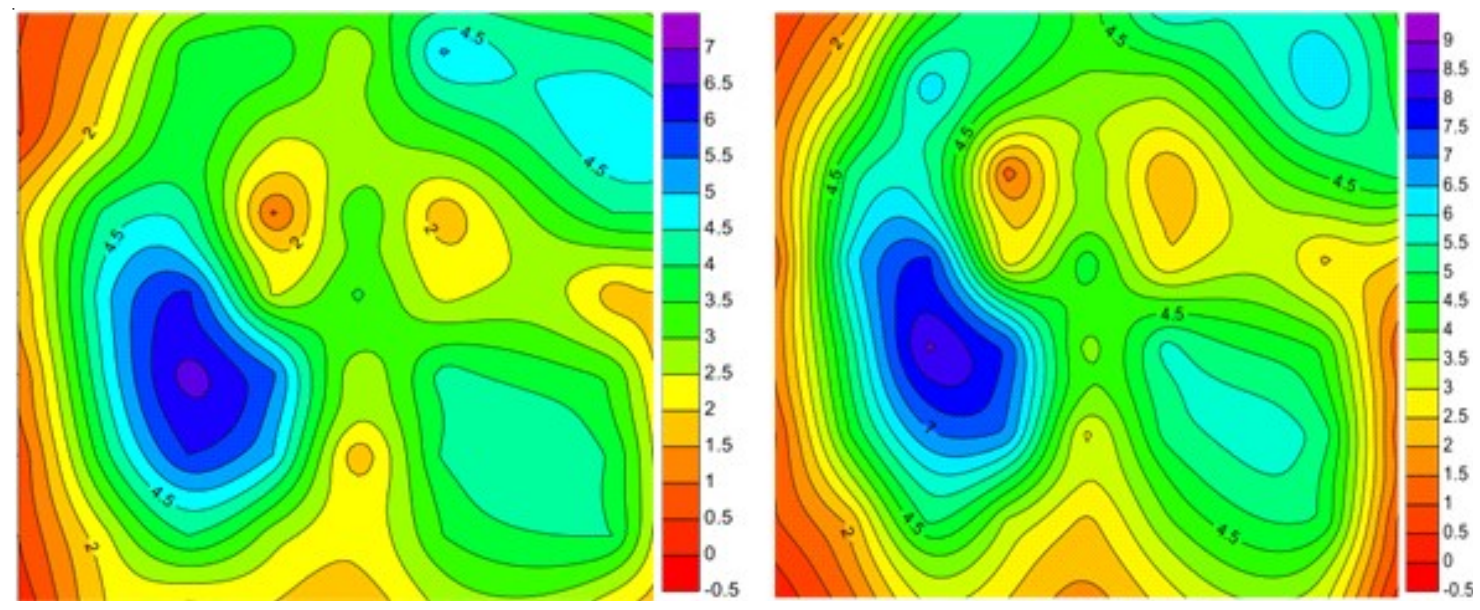

Fig. 5. . Distribución de velocidades de viento en la descarga del túnel de viento, a $70 \mathrm{~cm}$ de distancia, para (a) 20 y (b) 25 , Hz respectivamente. 
La capacidad de captación de la turbina eólica de acuerdo con la velocidad del viento se muestra en la Fig. 7, tomada del manual de operación para la turbina [21]. La velocidad está expresada en millas por hora $(\mathrm{mph})$ y la conversión a $(\mathrm{m} / \mathrm{s}=$ $0.45 \mathrm{mph})$.

Las curvas muestran un ancho de banda de potencia para un valor específico de velocidad. La curva inferior es para un perfil de viento ideal y de acuerdo con el nivel de turbulencia del viento, se incrementa hasta la curva superior, según el fabricante. Para la curva inferior se obtiene el máximo nominal de $12 \mathrm{~m} / \mathrm{s}=28 \mathrm{mph}$, que produce $400 \mathrm{~W}$.

$\mathrm{Al}$ eje se acopla un generador-alternador trifásico de imanes permanentes sin escobillas, a la salida de este un circuito rectificador para convertir la corriente alterna a corriente directa y un circuito de control para limitar la captación de energía mediante un freno por histéresis, la acción del cual, se puede apreciar en la Fig. 7, al disminuir la potencia drásticamente después de cierta velocidad. Cabe resaltar que la recomendación del fabricante es que la turbina se conecte a una batería junto con la carga para evitar la intermitencia.

El frenado por histéresis bloqueará la turbina cuando las baterías hayan alcanzado los $14.1 \mathrm{~V}$, y reanudará la carga cuando el voltaje esté por debajo de los $12.75 \mathrm{~V}$. Cuando el voltaje de la batería coincide con el punto establecido de regulación, la velocidad disminuirá drásticamente (a casi cero rpm) y la producción se detendrá. La operación de carga normal se reanudará cuando el voltaje de la batería caiga ligeramente por debajo del nivel completamente cargado.

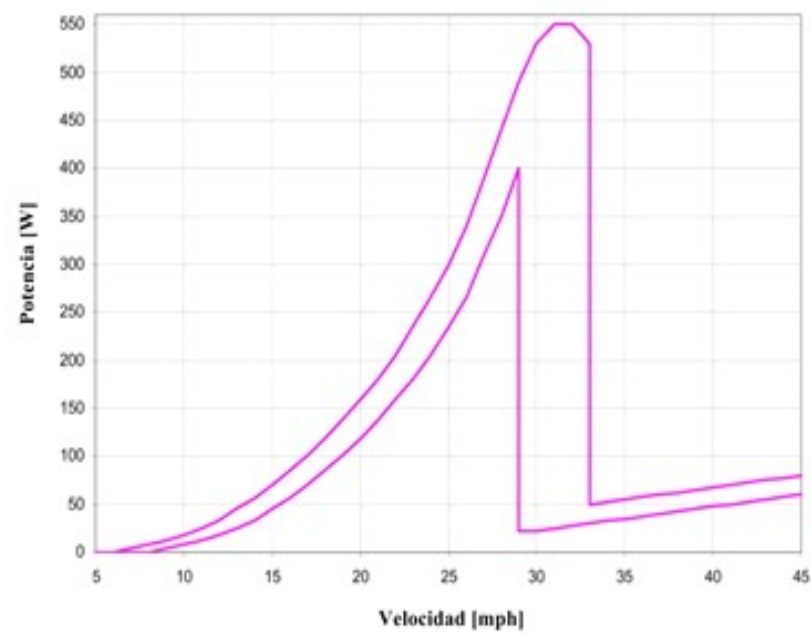

Fig. 7. Potencia de salida del aerogenerador, por la velocidad del viento en los álabes.

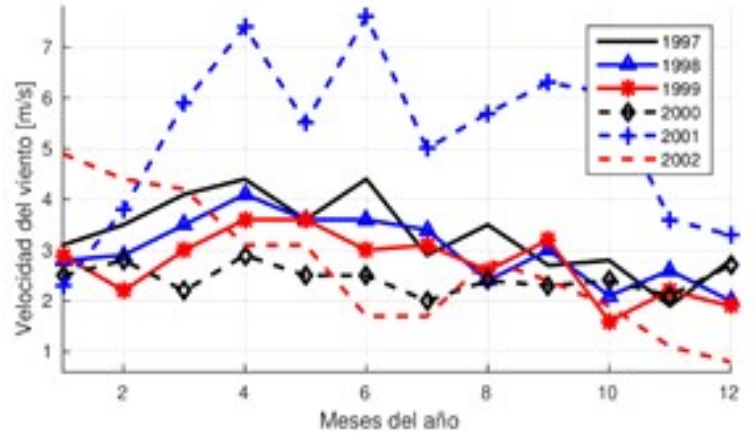

Fig. 8. Medición de velocidad del viento 1997-2002.

\subsection{Recurso eólico local}

De investigaciones en los medios locales, es interesante destacar que Quintana Roo es mencionado en 2014 por la evaluación de ProMéxico, entre algunos estados con recurso eólico potencial en México tales como Oaxaca, Baja California, Nuevo León, Tamaulipas y Veracruz [22]. Por otro lado, en [23] se menciona que para el funcionamiento de los parques eólicos se necesitan vientos de $7 \mathrm{~m} / \mathrm{s}$ en promedio y Quintana Roo no los mantiene para la mayor parte del año.

Además, en [9] se describe un estudio del recurso eólico en toda la península de Yucatán (del 2000 al 2007), mediante las estaciones de medición del Sistema Meteorológico Nacional (SMN). Cabe resaltar, que el promedio de la velocidad del viento en Chetumal no supera los $10 \mathrm{~m} / \mathrm{s}$.

En el presente trabajo se tiene registro de la velocidad del viento en la ciudad de Chetumal. En las Figs. 8 y 9 se muestran los perfiles históricos de velocidad del viento promedio para los 12 meses del año, desde 2009 hasta 2017. Los datos

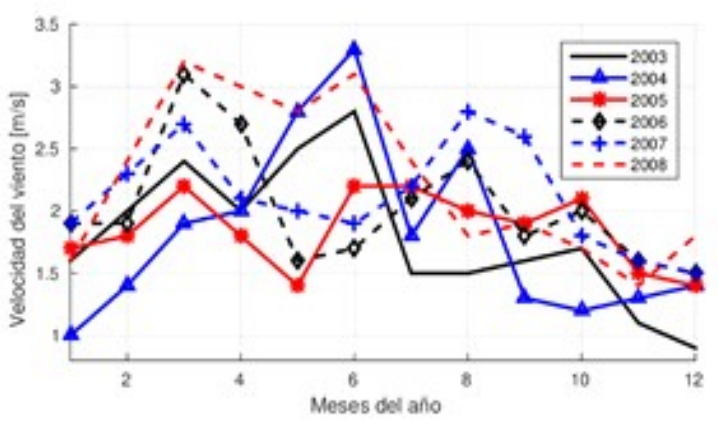

Fig. 9. Medición de velocidad del viento 2003-2008. 


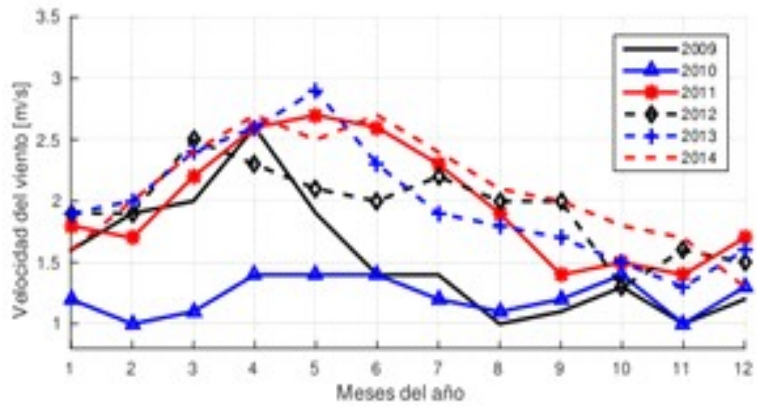

Fig. 10. Medición de velocidad del viento 2009-2014.

fueron obtenidos por la estación meteorológica instalada en el aeropuerto de Chetumal (misma de [9]) coordenadas $18^{\circ} 30$ $00200 \mathrm{~N} 88^{\circ} 1904000 \mathrm{~W}$. Se debe aclarar que los números de meses del año pertenecen a la numeración habitual, es decir, a enero le corresponde el número 1 , febrero el número 2 y así sucesivamente.

De la Fig. 8 a Fig. 11 se muestran los perfiles históricos de velocidad del viento para los 12 meses del año, desde 1997 hasta 2017. Los datos fueron obtenidos por la estación meteorológica instalada en el aeropuerto de Chetumal.

La Fig. 8 muestra los perfiles de la velocidad del viento para los años 1997 a 2002, donde se puede notar que para el 2001 el perfil de velocidad de viento es mayor que para el resto de los años mostrados.

En la Fig. 9 y la Fig. 10, se observa que la velocidad máxima promedio no sobrepasa los $3.5 \mathrm{~m} / \mathrm{s}$, y para todos los años se mantiene alrededor de $2 \mathrm{~m} / \mathrm{s}$. En la Fig. 10 se puede apreciar que la forma para los perfiles es muy similar para los años 2011 a 2014 y para el año 2010 un perfil muy bajo que oscila entre 1 y $1.5 \mathrm{~m} / \mathrm{s}$.

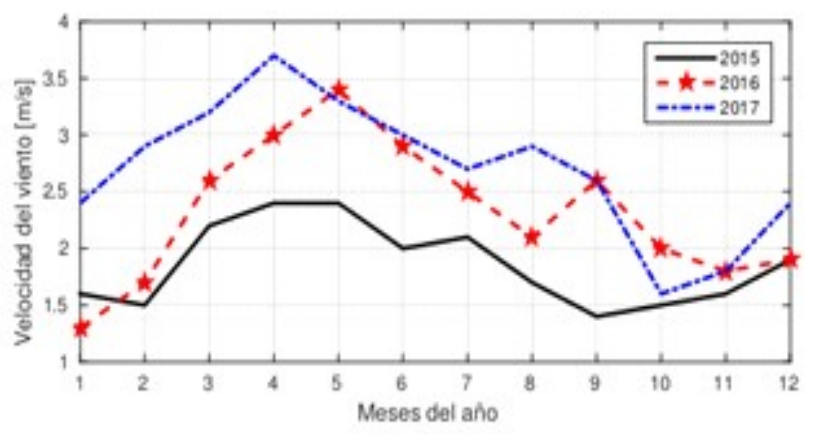

Fig. 11. Medición de velocidad del viento 2015-2017.
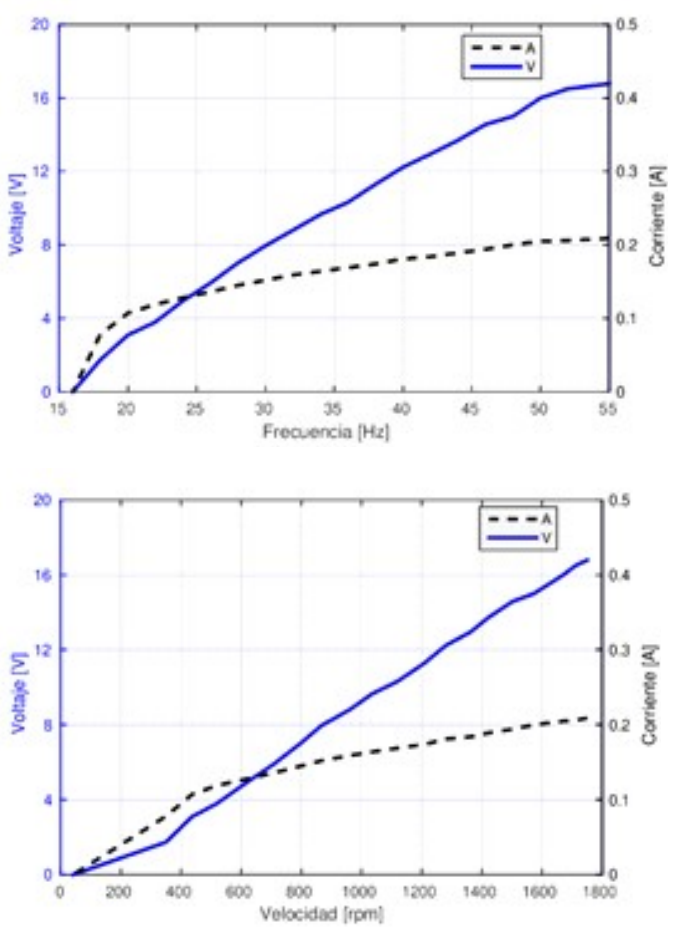

Fig. 12. Voltaje y corriente en la carga de $15.8 \Omega$, ante la variación de frecuencia en el túnel de viento y velocidad del aerogenerador.

De la Fig. 11 también es importante destacar que para los meses de marzo (3), abril (4) y mayo (5) en los tres años, se tienen velocidades promedio mayores que el resto del año. Por otro lado, de acuerdo con la velocidad promedio, se observa que para el año 2015 no se llega a $2.5 \mathrm{~m} / \mathrm{s}$, en 2016 , con una tendencia mayor no se alcanzó los $3.5 \mathrm{~m} / \mathrm{s}$, mientras que para 2017 apenas rebasa ese valor en el mes 4 (abril), respectivamente.

\section{Experimentos}

Se realizaron pruebas con la turbina eólica Air X $400 \mathrm{~W}$, colocándola en la azotea del edificio. Sin embargo, por la velocidad del viento que se tiene en la región, las tendencias mostradas en la sección anterior y la capacidad de la misma, el aerogenerador se mueve ligeramente, con dificultad y en muy pocos instantes de tiempo, lo que prácticamente no genera energía eléctrica.

Por lo anterior, se experimentó con el túnel de viento y el aerogenerador, colocando diferentes cargas (resistencias) y modificando las distancias entre el túnel de viento y la turbina de viento. Primero para una carga fija de $15.8 \Omega$, se modificó la frecuencia de trabajo para el moto-ventilador y con ello variar la velocidad del aerogenerador, la Fig. 12 muestra el 
comportamiento del voltaje y corriente en la carga menciona$\mathrm{da}$, ante la variación de frecuencia en el controlador del motoventilador y por ende cambios de velocidad en la turbina de viento.

Se midió el viento a la salida del túnel donde se colocó el aerogenerador, mediante un anemómetro para identificar el aire que llega a la turbina. Los mapas de la Fig. 13 se obtuvieron al variar la velocidad del viento en el túnel, mediante la frecuencia del motor, que a su vez varía la velocidad de giro del ventilador que extrae el aire. En esta medición se tomó un radio de $60 \mathrm{~cm}$, para captar toda el área que cubre el aerogenerador. Cabe resaltar que las velocidades del viento a la salida en general son turbulentas, como es lo esperado en estos sistemas no controlados, como se muestra también en [25].
Por último, la Fig. 14 muestra el comportamiento de las variables eléctricas voltaje $(V)$ vs. corriente $(I)$, para diferentes valores de carga $(R)$ y distancia $(D)$, donde se nota que se obtiene más energía de colocar a $70 \mathrm{~cm}$ de distancia entre el túnel y el aerogenerador $(D=70 \mathrm{~cm})$, con la menor resistencia $R=2.88 \Omega$ y conectando una batería de $12 \mathrm{~V}$ en paralelo.

\section{Conclusiones}

Se evaluó un aerogenerador a diferentes velocidades de viento controladas, así como la evaluación del mapa de distribución de viento a la salida del túnel de viento. El aerogenerador se colocó a diferentes distancias del túnel, para lograr la mayor captación de energía eléctrica y se modificó la resistencia de carga para analizar la generación eléctrica.

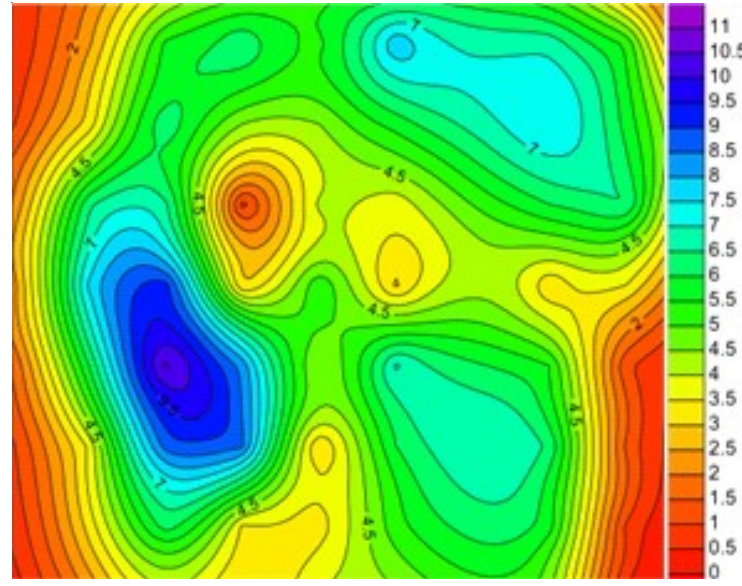

(a)

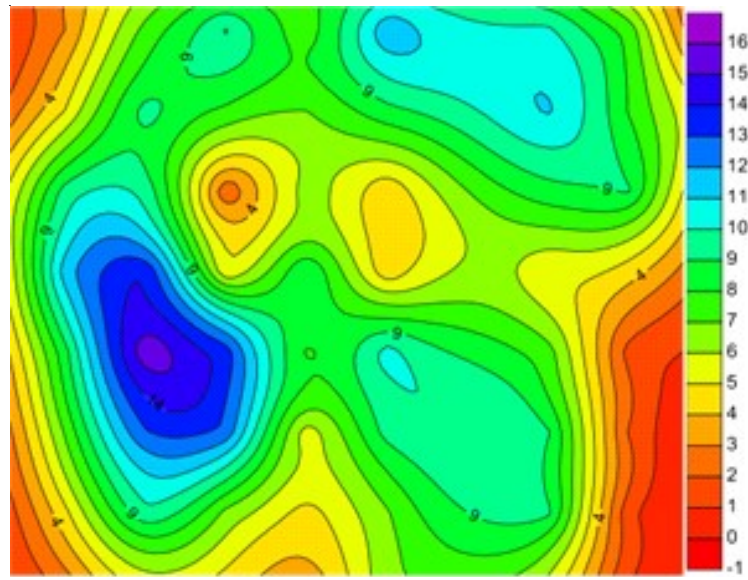

(a)

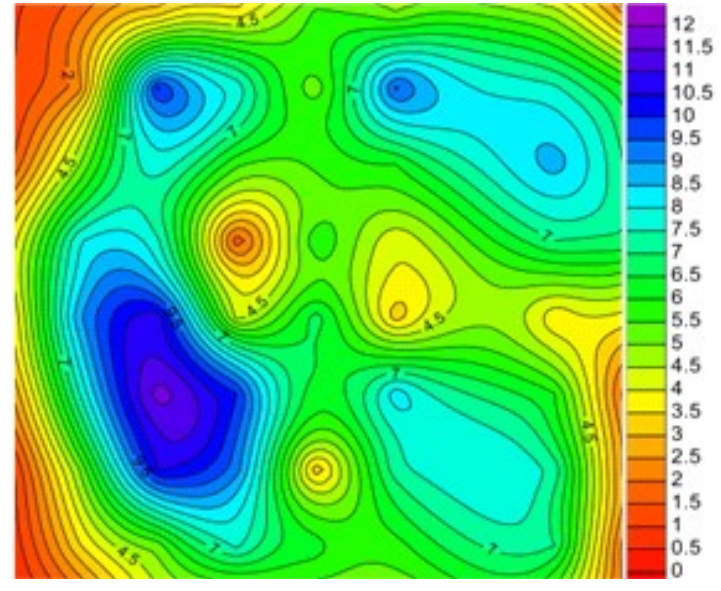

(b)

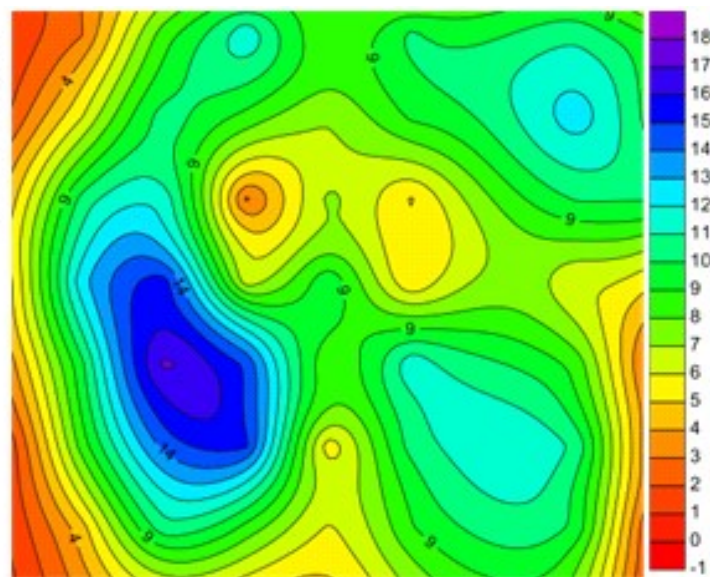

(b)

Fig. 13. Distribución de velocidades de viento en la descarga del túnel de viento a $70 \mathrm{~cm}$ de distancia. Para (a) 35, (b) 40 , (c) 45 y (d) $50 \mathrm{~Hz}$, respectivamente, en el variador de frecuencia del moto-ventilador del túnel. 


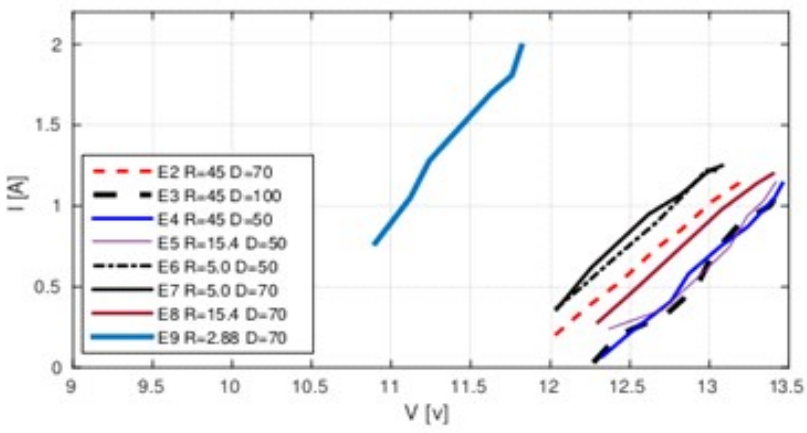

Fig. 14. Salida eléctrica del aerogenerador para diferentes resistencias y distancia del túnel.

Al colocar el aerogenerador fuera del túnel de viento, se logró aproximar a un sistema más real, a pesar de que el experimento se realizó en laboratorio, ya que la velocidad de viento en el ambiente exterior, en general, no se mantiene constante. Sin embargo, la medición del mapa de viento a la salida del túnel proporciona idea de la cantidad de energía eólica que se ingresa a la mencionada turbina de viento.

Mediante los experimentos es posible determinar el $C_{p}$ de operación de la turbina, con lo que será posible analizar el aerogenerador a escala, con perfiles de viento de la región y con el mismo recurso eólico determinar qué tipo de aerogenerador es más conveniente.

Para el mejor aprovechamiento del recurso eólico local, las recomendaciones principales para producir energía eléctrica son utilizar turbinas eólicas de eje vertical con buen desempeño a baja velocidad.

\section{Nomenclatura}

$\begin{array}{ll}P & \text { Potencia } \\ \rho & \text { Densidad del aire } \\ C_{p} & \quad \text { Coeficiente de potencia } \\ \lambda & \quad \text { Relación de velocidad de la punta del álabe } \\ \alpha & \text { Ángulo de inclinación del álabe } \\ A_{R} & \text { Área cubierta por los álabes durante la rotación } \\ R & \text { Radio del álabe } \\ \omega & \text { Velocidad angular del rotor de la turbina } \\ V_{v} & \text { Velocidad del viento } \\ \lambda^{\prime} & \text { Velocidad relativa normalizada } \\ r & \quad \text { Radio equivalente } \\ V_{m} & \text { Velocidad media del viento } \\ P_{v} & \text { Potencia extraíble del viento }\end{array}$

$\begin{array}{ll}P_{n} & \text { Potencia nominal } \\ V_{c i} & \text { Velocidad de arranque } \\ V_{n} & \text { Velocidad nominal } \\ V_{c f} & \text { Velocidad de paro } \\ A_{T} & \text { Área de trabajo del túnel } \\ A_{A} & \text { Área del aerogenerador }\end{array}$

\section{Rererencias}

[1] J. Chaves Palacio, "Desarrollo tecnológico en la primera revolución industrial," Revista de Historia, vol. 17, pp. 1-12, 2004.

[2] M. Best, A. Brown, P. Clark, D. Hollis, D. Middleton, G. Rooney, D. Thomson, C. Wilson, Small scale wind energy technical report. urban wind energy research project, Technical report, 2008.

[3] Y. Muñoz-Jadán, M. Espinoza-Bolaños, F. DonosoMerlet, R. Hidalgo-León, G. Soriano-Idrovo, P. JácomeRuiz "Hardware-in-the-Loop for Wind Energy Conversion with Resonant Current Control and Active Damping," IEEE Latin America Transactions, vol. 17, núm. 7, pp. 1146-1154, julio, 2019

[4] D. Elliott, M. Schwartz, G. Scott, S. Haymes, D. Heimiller, R. George. Wind energy resource Atlas of Oaxaca, National Renewable Energy Laboratory, Oak Ridge, 2003. TN 378310062.

[5] O. Jaramillo, M. Borja, "Wind speed analysis in la ventosa, mexico: a bimodal probability distribution case," Renewable Energy, Vol. 29, pp. 1613-1630, 2004.

[6] W.P. COMPLEX, 2018, Oaxaca ii-iii-iv wind power complex, disponible en https://www.accionaenergia.com/areas-of-activity/wind-power/majorprojects/oaxaca-ii-iii-iv-wind-power-complex/.

[7] L. Fried. Global wind energy statistics 2017. TR 1, Global Wind Energy Council, Brussels, Belgium 2018.

[8] C. Trust, Small scale wind energy, Technical report, Carbon Trust and Met Office. 2008.

[9] R. Soler-Bientz, S. Watson, D. Infield, "Wind characteristics on the yucatán peninsula based on short term data from meteorological stations," Energy Conversion and Management, vol. 51, pp. 754 - 764, 2010.

[10] Q. Hernández-Escobedo, F. Manzano-Agugliaro, A. Zapata-Sierra, "The wind power of Mexico," Renewable and Sustainable Energy Reviews, vol. 14, pp. 2830-2840, 2010.

[11] J. Mendoza-Vizcaino, A.Sumpera, A. Sudria-Andreu, J.M. Ramirez, "Renewable technologies for generation systems in islands and their application to Cozumel Island, Mexico," Renewable and Sustainable Energy Reviews, Vol. 64,pp. 348-361, octubre, 2016. 
[12] G. Hernández-Galvez, R. Saldaña, U. Miranda, O. Sarracino, M. Castillo, D. Almenares, A. K. Tapia-Gómez, "Wind resource assessment and sensitivity analysis of the levelised cost of energy. A case study in Tabasco, Mexico," Renewable Energy Focus, vol. 29, pp. 94106, junio, 2019.

[13] S. Heier, Grid Integration of Wind Energy Conversion Systems, Wiley, 2a ed., 2006.

[14] J. Villanueva, L. Alvarez-Icaza, Wind turbine modeling by friction effects, In the 17 th World Congress The International Federation of Automatic Control, 20082013. Seoul, Korea, 2008.

[15] Z. Lin, G. Qingding, Adjustable-pitch and variable speed control of wind turbines using non linear algorithm, In Sixth International Conference on Electrical Machines and Systems, Portland, OR, USA, pp. 270-273, 2003.

[16] M. Balas, A. Wright, M. Hand, K. Stol, Dynamics and control of horizontal axis wind turbines, In the 39th IEEE Conference on Decision and Control, USA, pp. 3781-3793, 2003.

[17] A. Dadone, L. Dambrosio, "Estimator based adaptive fuzzy logic control technique for a wind turbine generator system," Energy Conversion and Management, vol. 44, núm. 1, pp. 135-153, 2003.

[18] A. Maleki, F. Pourfayaz, "Optimal sizing of autonomous hybrid photovoltaic/wind/battery power system with lpsp technology by using evolutionary algorithms," Sol. Energy, vol. 115, pp. 471-483, 2015.
[19] H. Tazvinga, B. Zhu, X. Xia, "Optimal power flow management for distributed energy resources with batteries," Sol. Energy, vol. 102, pp. 104-110, 2015.

[20] M. Amrollahi, S. Bathaee, "Techno-economic optimization of hybrid photovoltaic/wind generation together with energy storage system in a stand-alone micro-grid subjected to demand response," Appl. Energy, vol. 202, pp. 66-77, 2017.

[21] L.Y. Villagrán-Villegas, I.V. Hernández-Rodríguez, J.C. Anzelmetti-Zaragoza, A. Marquina-Chávez, C. M. Calderón-Ramón, "Generalidades de estrategias de control en aerogeneradores de transmisión directa," Cientifica, vol. 21, núm. 1, pp 17-24, enero-junio 2017.

[22] Southwest Wind Power, Manual AIR-X, Air X The New 400 watt turbine, Flagstaff, Arizona 86001, octubre, 2002 .

[23] S. Blanco, Quintana Roo tiene potencial para energía eólica, disponible en: https://sipse.com/novedades/qroo-entre-los-cinco-estados-con-fuertes-corrientes-deviento-90087.html. 2014.

[24] A. Galicia, Carece Quintana Roo de potencial para parques eólicos, disponible en https://sipse.com/ novedades/carece-q-roo-de-potencial-para-parqueseolicos-energia-renovable-medio-ambiente-efectoinvernadero-cop13-cancun-233450.html. 2016.

[25] T. Fernández-Roque, J. A. Correa-Arredondo, J. F. Vázquez-Flores, J. Sandoval-Lezama, A. Mejía-Carmona, "El chorro en toberllino y los vórtices de punta de ala," Científica, vol. 21, núm. 2, pp 99-102, julio-diciembre 2017. 
